\title{
Toward on-chip, in-cell recordings from cultured cardiomyocytes by arrays of gold mushroom-shaped microelectrodes
}

\section{Anna Fendyur and Micha E. Spira*}

The Alexander Silberman Life Sciences Institute and the Harvey M. Kruger Family Center for Nanoscience, The Hebrew University of Jerusalem, Jerusalem, Israel

Edited by:

Michele Giugliano, University of

Antwerpen, Belgium

\section{Reviewed by:}

Michela Chiappalone, Italian

Institute of Technology, Italy

Fabrice O. Morin, Fatronik-Tecnalia

Foundation, Spain

*Correspondence:

Micha E. Spira, Department of Neurobiology, The Hebrew

University of Jerusalem, Givat-Ram Campus, Jerusalem 91904, Israel.

e-mail:spira@cc.huji.ac.il
Cardiological research greatly rely on the use of cultured primary cardiomyocytes (CMs). The prime methodology to assess CM network electrophysiology is based on the use of extracellular recordings by substrate-integrated planar Micro-Electrode Arrays (MEAs). Whereas this methodology permits simultaneous, long-term monitoring of the $\mathrm{CM}$ electrical activity, it limits the information to extracellular field potentials (FPs). The alternative method of intracellular action potentials (APs) recordings by sharp-or patch-microelectrodes is limited to a single cell at a time. Here, we began to merge the advantages of planar MEA and intracellular microelectrodes. To that end we cultured rat $\mathrm{CM}$ on micrometer size protruding gold mushroom-shaped microelectrode (gM $\mu \mathrm{Es}$ ) arrays. Cultured $\mathrm{CMs}$ engulf the gM $\mathrm{E}$ permitting FPs recordings from individual cells. Local electroporation of a $\mathrm{CM}$ converts the extracellular recording configuration to attenuated intracellular APs with shape and duration similar to those recorded intracellularly. The procedure enables to simultaneously record APs from an unlimited number of CMs. The electroporated membrane spontaneously recovers. This allows for repeated recordings from the same $\mathrm{CM}$ a number of times $(>8)$ for over 10 days. The further development of $\mathrm{CM}-\mathrm{gM} \mu \mathrm{E}$ configuration opens up new venues for basic and applied biomedical research.

Keywords: cardiomyocyte electrophysiology, electroporation, multi-electrode array, field potential, action potential

\section{INTRODUCTION}

Multiunit, non-invasive extracellular recordings by substrate integrated planar Micro-Electrode Arrays (MEAs) is currently the prime electrophysiological methodology for long-term electrophysiological analysis of cultured cardiomyocyte $(\mathrm{CM})$ activity. These MEAs serve as a platform for screening drugs, and elaborating therapeutic strategies (Sanchez-Bustamante et al., 2008; Yeung et al., 2009; Matsa et al., 2011; Law et al., 2012), the development of personalized medicine (Itzhaki et al., 2011) and may be instrumental in evaluating the use of cultured cells for functional integration with damaged heart tissue (Sekine et al., 2011). Similar planar MEAs are also used to interface cultured neurons or for brain-machine interfaces (Fromherz, 2006; Nicolelis and Lebedev, 2009; Jones et al., 2011). Monitoring CM electrical activity by planar MEA limits the electrophysiological information to extracellular field potentials (FPs) generated by action potentials (APs) in the vicinity of the electrodes. Detailed analysis of CM FP requires extensive computations, which rely on estimated parameters (Omura, 1970; Banach et al., 2003). On the other hand, the excellent signal-to-noise ratio provided by sharp-intracellular microelectrodes (Purves, 1981) and patch-electrodes (Sakmann and Neher, 1984) makes it possible to extract essential biophysical parameters underlying spike generation mechanisms and the properties of electrical synapses that couple different types of cardiac cells (Zipes and Jalife, 2009). Nevertheless, the use of sharp or patch microelectrodes is limited to a small number of cells, and because of continuous endogenous mechanical contraction, the friction between the rigid electrodes, and the cells leads to membrane rupture and cell death. In addition, patch-electrodes perfuse the cytoplasm and alter the intracellular composition of the cells thus limiting their use. To bypass the difficulties of using intracellular electrophysiological approaches to monitor electrical activity from contracting muscle cells, many laboratories now use voltage- or calcium-sensitive dyes (Herron et al., 2012). Although very effective for monitoring the beating rhythms, spread of APs and assessing the effects of drugs on these parameters, the loading of indicators may affect the electrophysiological properties of the cells (Nygren et al., 2003). Furthermore, imaging of the free intracellular calcium concentration kinetics reflects complex processes of calcium influx through voltage gated calcium channels, the release of calcium from intracellular stores, and the removal of calcium by a large number of mechanisms. Thus, currently the use of voltage sensitive dyes or calcium indicators cannot fully substitute for electrophysiological approaches.

To circumvent the limitations of planar-MEA, sharp- and patch-electrodes (as detailed above) and the side effects of various molecular probes, we began to explore in this study the use of micrometer size gold mushroom-shaped microelectrodes (gM $\mathrm{gEs}$ )-based MEA for intracellular recordings of APs from beating cultured rat CM. 
In earlier studies from our laboratory we developed the use of gM $\mu$ E-array to monitor intracellular synaptic and APs from cultured Aplysia neurons (Spira et al., 2007; Hai et al., 2009a,b, $2010 \mathrm{a}, \mathrm{b})$. The main principles underlying the neuron-gM $\mu \mathrm{E}$ interface were: the initiation of mechanisms by which cultured neurons actively engulf $\mathrm{gM} \mu \mathrm{Es}$ that protrude from the surface of the device (see Figure 1A for schematic representation), the formation of high seal resistance between the plasma membrane and the $\mathrm{gM} \mu \mathrm{Es}$ and the increased conductance of the plasma membrane facing the gM $\mu$ E's cap (Hai et al., 2009a,b). Our studies were followed by three additional reports that used nanofabricated pillars and field effect transistors to gain intracellular recordings from cultured cells (Duan et al., 2012; Robinson et al., 2012; Xie et al., 2012).

We report here that when coated by laminin (SanchezBustamante et al., 2008), the geometry of the gM $\mu$ Es is sufficient to promote the engulfment of the protruding $\mathrm{gM} \mu \mathrm{Es}$ by cultured CM (Figure 1), thus permitting gM $\mu$ Es with a relatively small surface area $\left(\sim 10 \mu \mathrm{m}^{2}\right)$ to record endogenously generated FPs by individual myocytes for many days. In response to a short electroporation pulse delivered through the gM $\mu \mathrm{Es}$, the functional $\mathrm{CM}-\mathrm{gM} \mu \mathrm{E}$ configuration is converted from an extracellular recording configuration to accessing intracellular recordings of attenuated APs. The shape and duration of the recorded APs is similar to those recorded intracellularly from rat CM. Autonomous recovery of the electropores generated at the plasma membrane facing the gM $\mu$ Es spontaneously revert the recording mode to extracellular. The device enables repeated transition from the extracellular to the intracellular mode of recordings over a number of days despite the continuous beating of the CM.

\section{METHODS}

\section{gM $\mu$ E-BASED MEA FABRICATION}

Arrays of gM $\mu \mathrm{E}$ electrodes for electrical measurements were prepared on $200 \mu \mathrm{m}$ thick glass wafers (AF45 Schott Glass) as previously described (Hai et al., 2009a, 2010b; Fendyur et al., 2011). Briefly, wafers were coated with a Ti $(10 \mathrm{~nm}) / \mathrm{Au}(100 \mathrm{~nm})$ layer by way of evaporation, spin-coated with photoresist AZ1505 (4000 RPM), and baked for $30 \mathrm{~min}$ at $90^{\circ} \mathrm{C}$ after which a first photolithographic process was performed to define the conduction lines by $\mathrm{Au} / \mathrm{Ti}$ wet etch. Next a second lithographic step
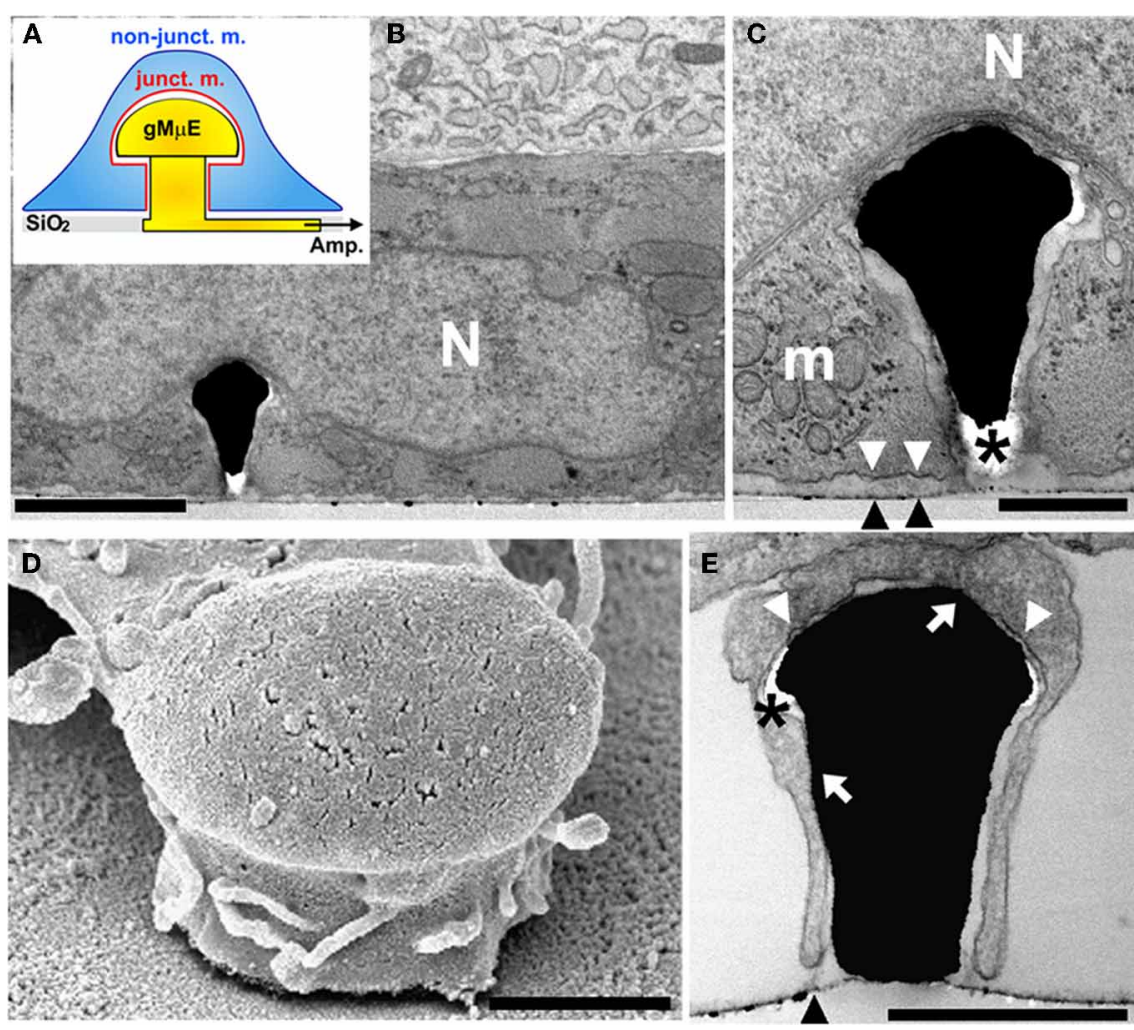

FIGURE 1 | A cultured cardiomyocyte engulfing a gM $\mu$ E. (A) Schematic representation of a $\mathrm{CM}$ (blue) engulfing a gM $\mu \mathrm{E}$ (yellow). Shown are the non-junctional membrane that faces the bathing solution (non-junct. $\mathrm{m}$. in blue) and the junctional membrane that faces the gold mushroom shaped microelectrode (junct. $\mathrm{m}$. in red). (B) A transmission electron-micrograph depicting a thin section through a $\mathrm{CM}$ engulfing a gM $\mu \mathrm{E}$. The base of the electrode is not seen in the plane of the section. (C) Enlargement of the extracellular space interfacing the cardiomyocyte plasma membrane and the "cap" of the gM $\mathrm{EE}$. (D) A scanning electron micrograph of a branch extending from a cultured cell partially engulfing a gM $\mathrm{EE}$. (E) A transmission electron micrograph of a delicate cell extension engulfing a gM $\mu \mathrm{E}$. $\mathrm{N}$-nucleus, $\mathrm{m}$-mitochondria, black arrow heads point to the culture substrate, white arrow heads point to the plasma membrane that faces the substrate, white arrows point to areas of close physical contact between the plasma membrane and the $\mathrm{gM} \mu \mathrm{E}, *$ depicts post fixation formed "holes" in the embedding material. Calibrations: $\mathbf{B}=2 \mu \mathrm{m}, \mathbf{C}=0.5 \mu \mathrm{m}, \mathbf{D}, \mathbf{E}=1 \mu \mathrm{m}$. 
using S-1813G2 photoresist was performed to open holes for the deposition of the gM $\mu \mathrm{E}$ stalks as well as the contact pads. Next the gM $\mu$ Es were formed by way of gold electroplating at a current density of $0.15 \mathrm{~A} / \mathrm{cm}^{2}$ for $15-20 \mathrm{~min}$. The photoresist layer was stripped off and a layer of silicon oxide ( 3000 Angstrom) was deposited by chemical vapor deposition. This layer serves to passivate the conducting electrode lines. A third layer of photoresist was then applied. A third lithographic step was used to expose the contact pads and the caps of the gold mushrooms followed by wet oxide etch to selectively remove the oxide from the contact pads and the mushroom caps. Retrospective SEM of the gM $\mathrm{Es}$ revealed that the oxide on the upper (third) part of the stalk was also etched. Wafers were then diced and underwent manual bonding to 62-pad printed circuit boards to which $21 \mathrm{~mm}$ glass rings were attached to create a recording bath chamber for the culturing medium.

\section{FABRICATION OF GOLD MUSHROOM SHAPED MICRO PROTRUSIONS MATRICES FOR ELECTRON MICROSCOPY}

Scanning- and transmission-electron microscopic imaging were conducted using dissociated cultured CMs grown on matrices of gold-mushroom shaped protrusions ( $\mathrm{gM} \mu \mathrm{P})$. The fabrication of gM $\mu \mathrm{P}$ matrices were prepared on $200 \mu \mathrm{m}$ glass wafers (AF45 Schott Glass) by means of photolithography and electroplating techniques, as described above.

The slides were attached to culture dishes using silicone (Sylgard Dow Corning).

\section{CELL CULTURE}

The culturing procedures followed the ethical guidelines of Care and Use of Laboratory Animals and were approved by the Committee for Animal Experimentation at the Institute of Life Sciences of the Hebrew University. Primary cultures of CM were prepared as previously described (Shneyvays et al., 1998). The hearts of 1 day old rats were removed, washed in phosphate-buffered saline (PBS), minced to small fragments, agitated in a solution of proteolytic enzymes (RDB, Biological Institute, Ness-Ziona, Israel). The cells were then diluted 1:50 in $\mathrm{Ca}^{2+}$ and $\mathrm{Mg}^{2+}$-free PBS at $25^{\circ} \mathrm{C}$. Several cycles of dissociated cardiac cell collection were performed lasting 10-15 min each. Dulbecco's modified Eagle's medium (DMEM) containing $10 \%$ horse serum (Biological Industries, Kibbutz Beit Haemek, Israel) was added to supernatant suspensions containing dissociated cells. The mixture was centrifuged at $300 \mathrm{~g}$ for $5 \mathrm{~min}$. The supernatant phase was discarded, and the cells were suspended again. The suspension of the cells was diluted to $1 \times 10^{6}$ cells $/ \mathrm{ml}$, which were plated on the laminin-coated $(20 \mu \mathrm{g} / \mathrm{ml})$ surfaces. The cultures were incubated in a humidified atmosphere of $5 \% \mathrm{CO}_{2}, 95 \%$ air at $37^{\circ} \mathrm{C}$. Confluent monolayers exhibiting spontaneous contractions were developed in culture within 2-3 days. The growth medium was replaced after $24 \mathrm{~h}$ and then every 3 days.

\section{ELECTRON MICROSCOPY}

For both SEM and TEM analysis cells cultured on the gold spine substrate were fixed, dehydrated, and embedded in Agar 100 within the culturing dish as previously described (Spira et al., 2003). Briefly, CM were fixed by 3\% glutaraldehyde in $0.1 \mathrm{M}$ cacodilate buffer, $\mathrm{pH}$ 7.4. The cells were then washed with $0.1 \mathrm{M}$ cacodilate buffer (Agar Scientific, Stansted, England). The cells were post-fixed in $1 \%$ osmium tetroxide (Next Chimica, Centurion, South Africa) and $0.8 \% \mathrm{~K}_{3} \mathrm{Fe}(\mathrm{CN})_{6}$. Dehydration was carried out through a series of ethanol solutions. For TEM the neurons were embedded in Agar 100 (Agar Scientific, Stansted, England). Then the glass substrate was etched using $30 \%$ hydrofluoric acid (for approximately $3 \mathrm{~h}$ ). The Ti/Au layer was partially etched by diluted Au etcher $\left(\mathrm{I}_{2} / \mathrm{KI} / \mathrm{H}_{2} \mathrm{O}\right)$ and diluted HF (1:40), leaving the gold spine structures intact. Thereafter, the agar block including the cells was re-embedded in Agar 100 in a flat mold. This doubly-embedded preparation was then thin-sectioned.

Measurements of cleft width from TEM images were done digitally using the image analysis program ImageJ (NIH, USA) as previously described (Fendyur et al., 2011). Each image was divided into three areas: (1) gold mushroom cap, (2) gold mushroom stalk, and (3) flat gold substrate in between the gold spines. The sampling locations were selected within a grid (100 nm pitch) randomly placed on the image. The distance between the cell membrane and the mushroom was measured along the corresponding fraction of the grid lines.

\section{ELECTROPHYSIOLOGY}

For recording and electroporation gM $\mathrm{EE}$-based MEA were used. In a series of preliminary experiments we selected the parameters for electroporation to be: positive square pulse duration of 50-100 ms and 0.5-1 V. The described experiments were then conducted in the following manner: we recorded spontaneous FP activity from the entire $\mathrm{gM} \mu \mathrm{E}$ array for a number of minutes. We next selected gM $\mu \mathrm{E}$ that revealed FPs of $\geq 250 \mu \mathrm{V}$ for electroporation. The amplitude of the applied electroporating pulse was gradually increased from $0.5 \mathrm{~V}$ in $100 \mathrm{mV}$ steps until electroporation was monitored. Electroporation occurred in an all or none fashion. For electroporation of a number of CMs in the same culture dish we selected gM $\mu$ Es that were at least $40 \mu \mathrm{m}$ apart. After electroporation recordings lasted a number of minutes after the recovery of the electroporated membrane as evident by the reappearance of a typical extracellular potential. Altogether we have conducted $>40$ electroporating experiments using 30 cultures. For studies of repeated electroporation over days the cultures were kept in the incubator in between the experimental sessions. The signals were amplified by an AC, 60-channel amplifier (MEA-1060-Inv-BC, MCS) with frequency limits of $1-10,000 \mathrm{~Hz}$ at a sampling rate of $50 \mathrm{kHz}$. Origin 8.1 software (OriginLab Corp., Northampton, MA, USA) was used to plot the results.

\section{RESULTS}

\section{STRUCTURAL RELATIONSHIPS BETWEEN THE CARDIOMYOCYTES AND THE GOLD MUSHROOMS MICROELECTRODE SUBSTRATE}

As the morphology and physiology of cultured rat CMs is influenced by the sub-micrometer topography and stiffness of the culturing substrate (for example Wang et al., 2011) we began the study by testing the compatibility of laminin coated gold-mushroom microstructures as a substrate for culturing 
dissociated rat CMs. To that end we compared the development of plated dissociated CMs (density of $1 \times 10^{6}$ cells $/ \mathrm{ml}$ ) on flat glass surfaces and on matrices of 1-2 $\mu \mathrm{m}$ gM $\mu$ Es fabricated on glass spattered by a thin film of gold. The inter-gM $\mu$ Es interval was $8 \mu \mathrm{m}$. Both substrates were coated by laminin. On both substrates the cells adhered and began to spontaneously contract within 2-3 days after plating.

We next processed beating cultured CM for transmission and scanning electron microscopic examination (TEM and SEM respectively). Structural analysis (of 4-6 day old cultured CM) revealed two cell types: cardiac muscle cells characterized by a typical acto-myosin filament packing pattern, dark glycogen particles, and mitochondria and fibroblasts that lack acto-myosin machinery (Figure 1). Many of the CMs and fibroblasts adhered to the flat substrate in between the gM $\mu \mathrm{E}$, and engulfed at least one gM $\mu \mathrm{E}$ (Figure 1). The averaged width of the extracellular cleft formed between adjacent CMs was in the range of $28.8 \pm 2.1$ (mean $\pm \mathrm{SE}$ ) nm [10 different CM-gM $\mathrm{CE}$ junctions were analyzed as previously described by us (Fendyur et al., 2011)]. Thirty-four percent of the CM-plasma membrane-gM $\mu$ Es junctions appeared to be in direct contact (defined as $0-5 \mathrm{~nm}$ cleft). Twenty-two percent of the junctional area revealed a space of 5-25 nm, $18 \%$ of the junctional area ranged between 25 and $50 \mathrm{~nm}, 19 \%$ in the range of $50-100 \mathrm{~nm}$, and $7 \%$ above $100 \mathrm{~nm}$. The extracellular space formed between the myocytes and the flat substrate (in-between the electrodes) was $98 \pm 5.7$ (mean $\pm \mathrm{SE}$ ) nm (Figure 1). Since laminin was homogeneously applied to the culture substrate it is reasonable to conclude that the geometry of the gM $\mathrm{E}$ s facilitated the formation of the reduced extracellular space between the gM $\mu$ Es and the cell's membrane.

Using SEM complemented by TEM observations we found that individual gM $\mu$ Es were often engulfed by thin protrusions that extend from the cell bodies (Figure 1). In some cases the thin branches adhered to the mushroom cap and to parts of the "mushroom's stalk" leaving other parts of the gM $\mu \mathrm{E}$ stalk in contact with the extracellular solution (see also SEM image Figure A1).

\section{EXTRACELLULAR FIELD POTENTIAL RECORDINGS BY THE GOLD MUSHROOM-BASED MEA}

The MEAs used in this study were composed of $8 \times 8 \mathrm{gM} \mu \mathrm{Es}$ (two of which were not bonded) with a cap diameter of $1-2 \mu \mathrm{m}$ and a pitch of $20 \mu \mathrm{m}$ covering an area of $147 \times 147$ $\mu \mathrm{m}\left(\sim 21,000 \mu \mathrm{m}^{2}\right)$. The gM $\mu$ Es and the flat glass substrate in between the gM $\mathrm{g}$ Es were functionalized by laminin. Because of the small dimensions of the gM $\mu$ Es and their pitch, a number of gM $\mathrm{E}$ Es were expected to be in physical contact with a single CM (with an estimated diameter of $\sim 40 \mu \mathrm{m}$ ) and only a few CM could be recorded from. Recordings and electroporation pulses were made by $62 \mathrm{gM} \mu \mathrm{Es}$ using the Multi Channel Systems (Reutlingen, Germany) AC amplifier (MEA1060 -Inv-BC), with frequency limits of $1-10,000 \mathrm{~Hz}$ and a gain of 110-1100. The data shown is of raw, unprocessed recordings. Typically, the background noise level of the system was $\sim 20 \mu \mathrm{V}$. In all experiments a $20 \mathrm{~ms} 1 \mathrm{mV}$ voltage calibration square pulse was applied to the bathing solution by an isolated pulse generator.
Recording of spontaneous electrical activity was made from 3 to 14 days old cultures. Altogether we recorded spontaneous field potentials from $>50$ cultures). Although the estimated surface area of a $\mathrm{gM} \mu \mathrm{E}$ is small $\left(\sim 10 \mu \mathrm{m}^{2}\right)$ the FPs recorded by individual $\mathrm{gM} \mu \mathrm{E}$ are very similar in amplitude and shapes to those reported earlier using larger substrate integrated planar electrodes (Banach et al., 2003; Reppel et al., 2005; Jones et al., 2011). This is consistent with the transmission electron micrographs indicating that the engulfment of the gM $\mathrm{E}$ Es by the cells increases the seal resistance formed between the cells and the electrode and thereby compensates for the relative smaller surface area of the electrodes (Figure 1; Hai et al., 2009a,b, 2010a,b; Fendyur et al., 2011). Most recorded FPs were composed of a positive phase representing the outward current generated by neighboring cells to the one from which the recording is made, followed by an inward phase that subsides to the baseline representing the upstroke phase of the AP and its repolarization to the resting level (Figures 2, 3 and 4; see Halbach et al., 2003).

\section{FROM EXTRACELLULAR TO INTRACELLULAR RECORDINGS}

Application of a single 50-100 ms long $0.5-1 \mathrm{~V}$ positive square pulse through a gM $\mu \mathrm{E}$ leads to a transition from typical biphasic extracellular recordings of FPs to an intracellular recording mode of attenuated APs ranging between 1 and $6 \mathrm{mV}$ (Figures 2, 3, 4, and 5, 30 cultures, $>40$ individual $\mathrm{CMs}$ ). The recorded potentials have the characteristic shape of cultured rat CM APs with a sharp rise time of $5-7 \mathrm{~ms}$ (rise times were measured between 10 and $90 \%$ of the AP's peak value), a slower decay time and an afterhyperpolarizing potential of $0.4-1.5 \mathrm{mV}$ (Halbach et al., 2003). The transition from extracellular to intracellular recording of APs is attributed to localized increased conductance of the plasma membrane facing the gM $\mu \mathrm{E}$ by electroporation (Figure 1A and see Xie et al., 2012).

The amplitude of the intracellularly recorded AP gradually diminishes over 2-10 min and reverts to extracellular FP recording mode (Figures 2, 3). The nature by which the reduction in the AP amplitude proceeds (Figure 3) suggests that it occurs by recovery of the electroporated nanopores at the junctional membrane (Figure 1A) and not because of pathological processes that reduce the driving forces of inward and outward ionic currents across the plasma membrane. In support of these conclusions we found that during a substantial phase from electroporation, the shape of the AP is maintained constant while its amplitude is diminished. This is illustrated in the experiment of Figure 3 in which normalized APs were superimposed revealing that the AP shape is not altered while the amplitude is reduced (Figures 3C,D). This behavior is consistent with a gradual reduction in the conductance of the electroporated patch of the plasma membrane that faces the electrode (schematically depicted in Figure 2C). If the attenuation of the APs had been the consequence of increase in the intracellular calcium and sodium concentrations the ensuing reduction in the driving forces of these ions, would have changed the shape of the APs. At late phases of the recovery process, when the amplitude of the intracellularly recorded AP is reduced to value of the FP (prior to electroporation) the shape of the recorded AP represent complex contributions of extracellular (FP) and intracellular (APs) 


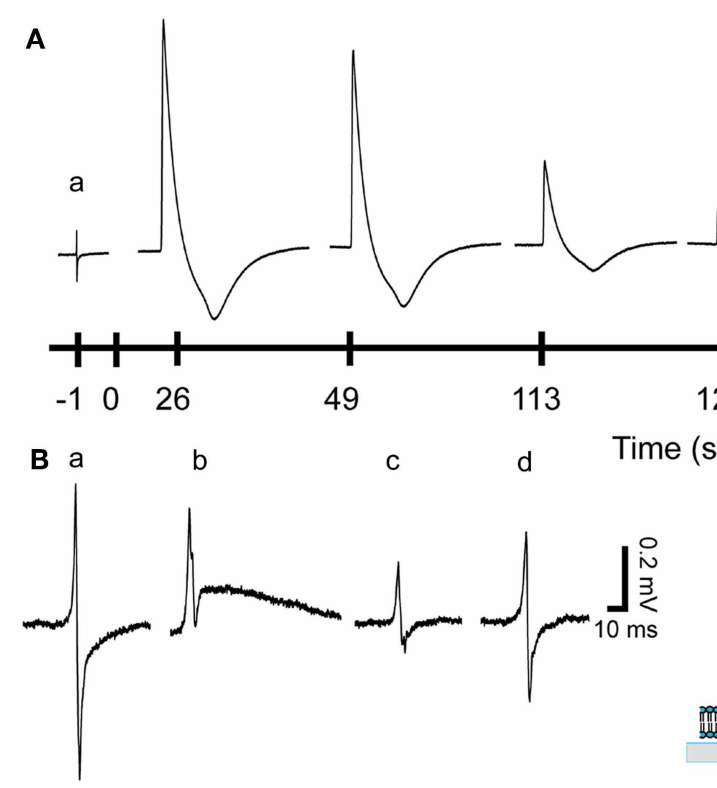

FIGURE 2 | From extracellular field potential (FP) recordings to intracellular recordings and the recovery process. $(\mathrm{Aa})$ Before electroporation a gM $\mu \mathrm{E}$ recorded a typical extracellular FP (enlarged in $\mathbf{B a}$ ). After the delivery of an electroporating pulse (100 ms $1 \mathrm{~V}$ ) (at $0 \mathrm{~s}$ ) the biphasic recorded $\mathrm{FP}$ transformed into a monophasic $5 \mathrm{mV}$ positive potential with a shape similar to that reported by intracellular recordings. The amplitude of the action potential diminished over time, gradually resuming the shape of the extracellular field potential 125-148s after electroporation. Thereafter the shape of the FP gradually recovered (between 148 and $607 \mathrm{~s}$ and enlarged in $\mathbf{B})$ regaining the typical biphasic shape (Ad and Bd). (B) Enlargements of the FPs before electroporation (Ba) and b $148, \mathbf{c} 607 \mathrm{~s}$ and $\mathbf{d} 24 \mathrm{~h}$ after electroporation. (C) Schematic drawing of the presumed reversible effects of an electroporating pulse on the plasma membrane facing a $\mathrm{gM} \mu \mathrm{E}$. (D) A calibration square pulse delivered to the bathing solution. currents (for example Figures $\mathbf{2} \mathbf{A b}, \mathbf{B b}$ ). The recovery rate was not correlated to the age of the cells in culture or the amplitude of the attenuated AP.

\section{MULTISITE INTRACELLULAR RECORDINGS}

Simultaneous recording of FPs from a number of cultured CM is often used to characterize the spreading of APs along electrically coupled CM and thereby to assess and pharmacologically manipulate the electrical coupling between the cells. Using the gM $\mu$ E-based MEA such observations can be made by simultaneous intracellular recordings from a number of CMs as depicted in Figure 4. For the experiment, an electroporating pulse was simultaneously delivered to $4 \mathrm{gM} \mu \mathrm{Es}$ that recorded extracellular FPs (Figure 4). This led to the transformation of the recording configuration from an extracellular to an intracellular mode (Figures $4 \mathbf{C a}, \mathbf{C b}$ ). It is important to note that other gM $\mu \mathrm{Es}$ through which an electroporating pulse was not delivered maintained their extracellular recording mode (Figure 4Be). Superpositioning of the FPs and the APs recorded by the four different electrodes (Figures $\mathbf{4 C a}, \mathbf{C b}$, respectively) revealed clear delays in the onset of the potentials, reflecting the network properties of these cultured myocytes.

\section{REPEATED ELECTROPORATION AND LONG-TERM RECORDINGS}

Another important feature of the gM $\mathrm{EE}$-based MEA is that cultured CM can be electroporated a number of times over a number of days. Figure 5 shows six out of eight consecutive extracellular and intracellular recordings made by the same
$\mathrm{gM} \mu \mathrm{E}$ (presumably from the same CM) over a period of 11 days.

\section{DISCUSSION}

In the present study we began to develop the use of gM $\mu \mathrm{E}$ based arrays for simultaneous intracellular recording of APs from many beating CMs. We report that: (a) the physical junction formed between cultured rat CM and laminin coated, micrometer sized gM $\mathrm{E}$ Es is sufficient to enable extracellular recording of FPs generated by individual myocytes for weeks and (b), that in response to a short electroporation pulse delivered through the $g M \mu E s$, the $C M-g M \mu E$ configuration is converted from an extracellular recording configuration to accessing intracellular recordings of attenuated APs. The shape and duration of the recorded APs is similar to those recorded intracellularly from rat CM. Autonomous recovery of the electropores generated at the plasma membrane facing the gM $\mu$ Es revert the recording mode to extracellular. The gM $\mu$ Es-based device enables repeated transition from the extracellular to the intracellular mode of recordings over a number of days despite the continuous beating of the CM.

\section{STRUCTURAL RELATIONSHIPS BETWEEN CULTURED CARDIOMYOCYTES AND gM $\mu \mathrm{E}$}

Earlier studies documented that cultured CM sense submicrometric substrate topographies and respond by morphological adaptation and cytoskeletal reorganization (for example Wang et al., 2011). Consistent with the earlier studies, we 


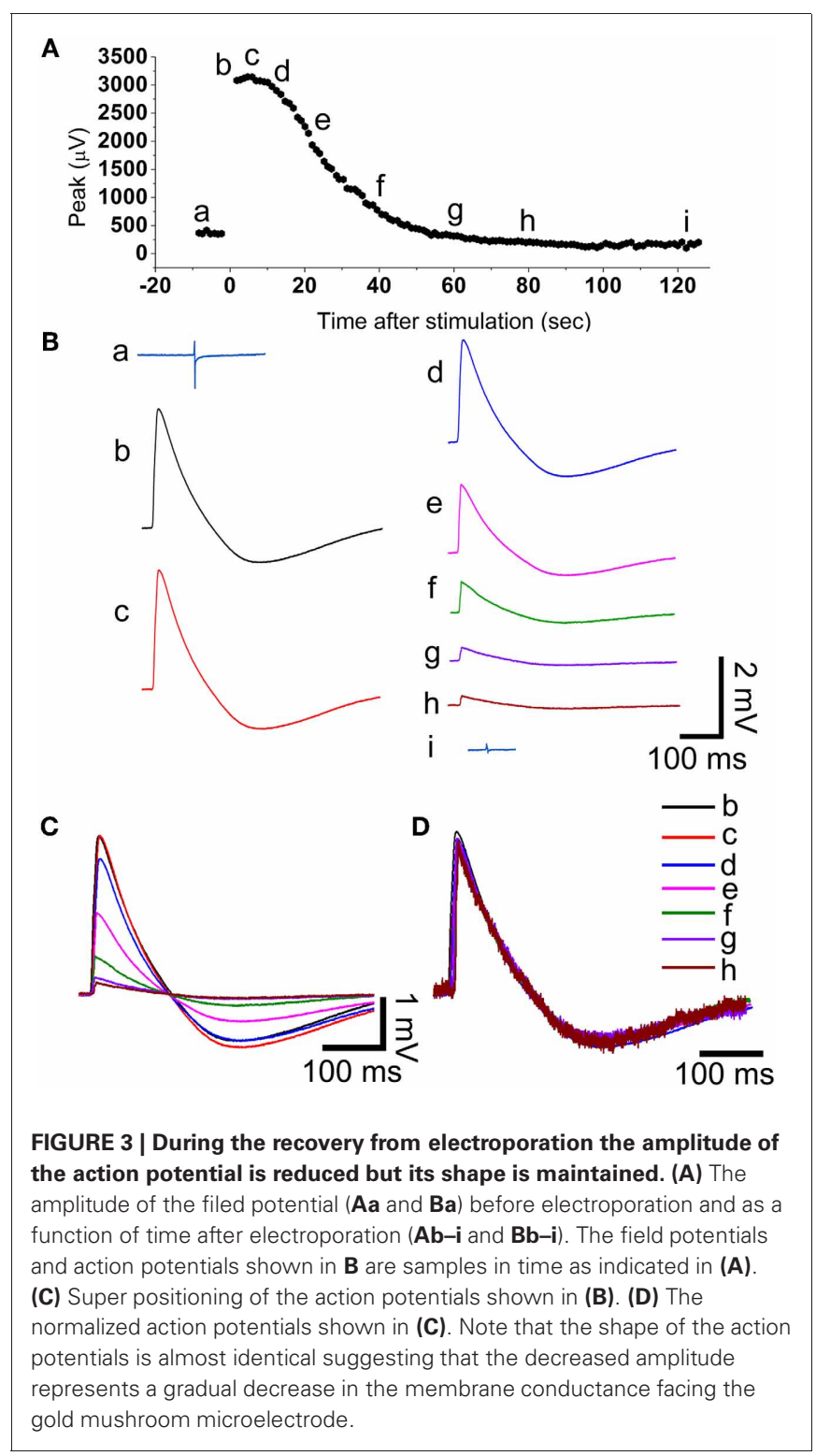

noted that the CM responded to the presence of the gM $\mathrm{gEs}$ by their engulfment. Further to that, we documented that $34 \%$ of the CM-plasma membrane-gM $\mathrm{CEs}$ junctions appeared to be in direct contact (defined as $0-5 \mathrm{~nm}$ cleft) with the $\mathrm{gM} \mu \mathrm{E}$. Interestingly, thin branches that extend from the cells also engulf the $\mathrm{gM} \mu \mathrm{E}$ and form a junction of close membrane apposition. Assessment of the dimensions of the extracellular cleft formed between the CM plasma membrane and the gM $\mu$ E from TEM micrographs must take into consideration the possibility that the chemical fixation, dehydration, and embedding might generate alterations in the intracellular osmotic pressure and thereby generate structural artifacts (Studer et al., 2008). Our analysis revealed that the narrowest junctions were consistently formed between the $\mathrm{CM}$ membrane and the gM $\mu \mathrm{E}$ cap rather than with the flat substrate in between the protruding $\mathrm{gM} \mu \mathrm{E}$. It is conceivable that the engulfment of the 3-dimensional structure, by

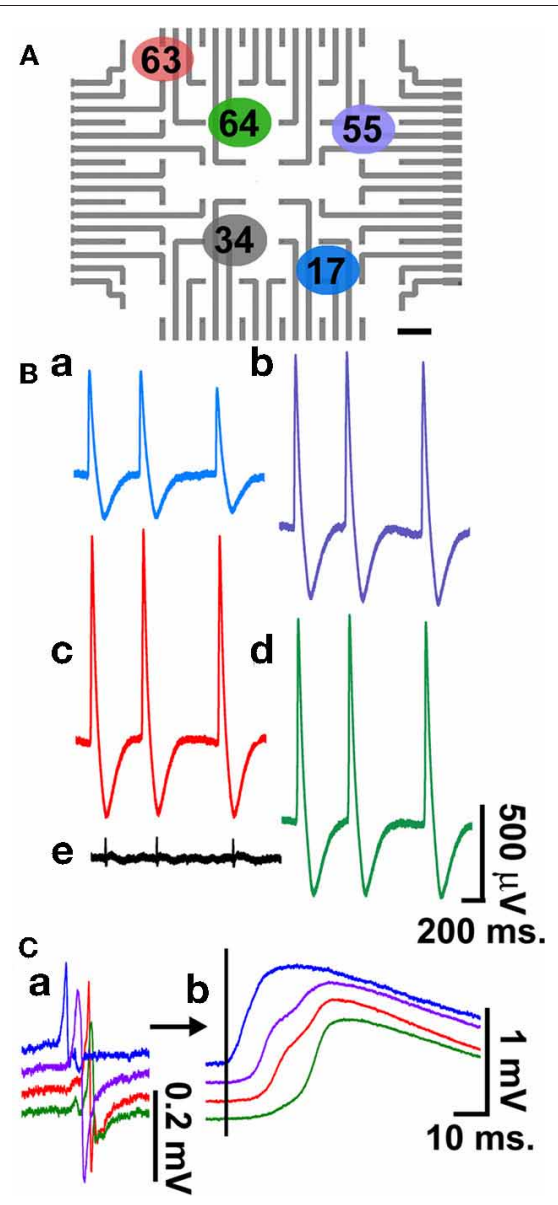

FIGURE 4 | Simultaneous multi-CM recordings. Recordings of intracellular action potentials and FPs generated by electrically coupled, beating cultured $\mathrm{CM}$ grown on laminin functionalized gM $\mu$ Es-based MEA for 4 days. For the experiment an electroporating pulse was delivered to electrodes 17(a), 55(b), 63(c), and 64(d), (A). Recordings are shown from these electrodes and from electrode 34(e), which was not electroporated (B). Superpositioning of the FPs prior to electroporation (Ca) and intracellular action potentials (b) reveals a $6-8 \mathrm{~ms}$ delay in the onset of the depolarization consistent with the propagation of the electrical impulse between the cells.

the aid of actin and other submembrane cytoskeletal elements generates mechanical tension around the curving geometry of the $\mathrm{gM} \mu \mathrm{E}$ and that this mechanical tension may underlie the formation of a narrow cleft in-between the plasma membrane the gM $\mu \mathrm{E}$ cap and stalk. Nevertheless, there exists a possibility that the tight apposition formed at this site may be the outcome of a fixation artifact. The well preserved subcellular organelles in the electron micrographs, suggest that the fixation, dehydration, and embedding procedures did not produce osmotic pressure artifacts. It is also important to note that the presence of electron translucent breaks within the embedding material, mainly at the curving junctions between the cells and the $\mathrm{gM \mu E}$ cap as well as the stalk (Figure 1), suggests that mechanical tension generated within the embedding polymer (Agar 100) lead to the detachment of the plasma membrane from 


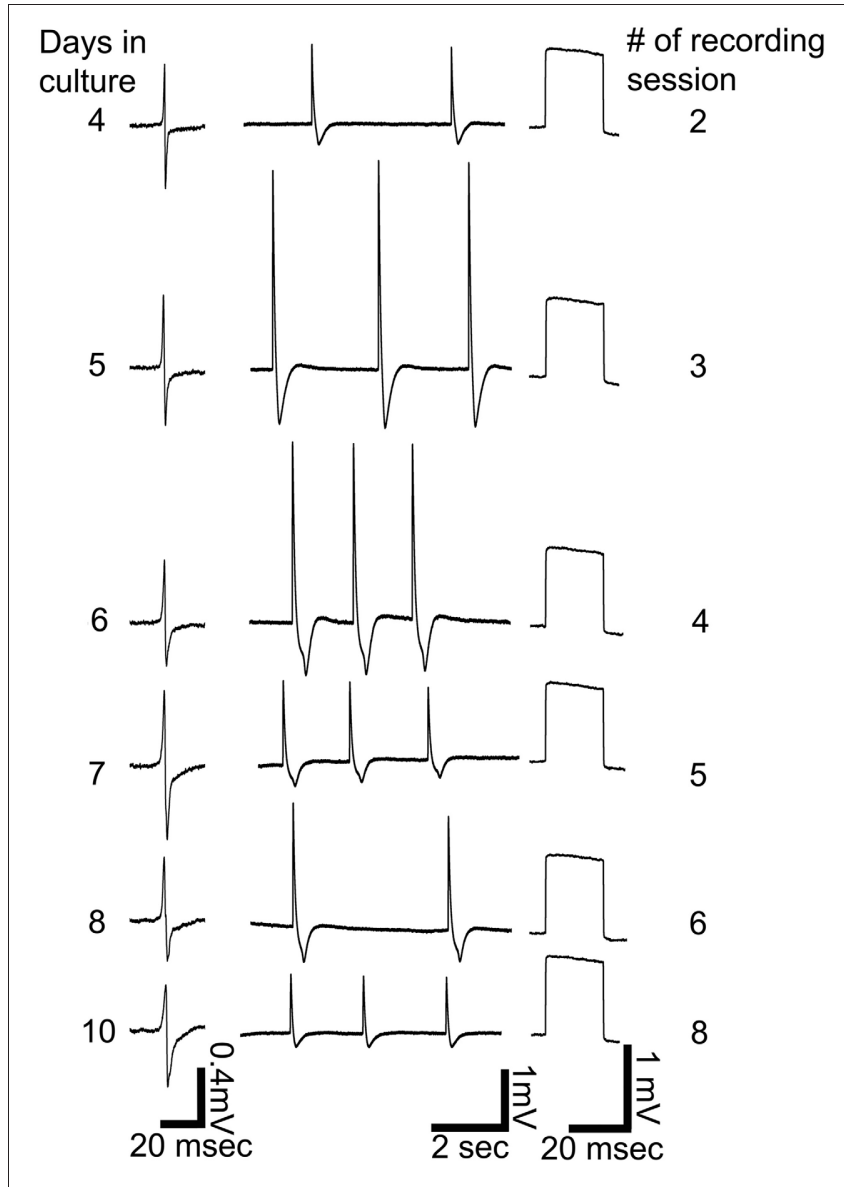

FIGURE 5 | Repeated intracellular recordings by a single $\mathrm{gM} \mu \mathrm{E}$ on days $4,5,6,7,8$, and 10 after plating. Left column, extracellular field potentials before electroporation, middle column, spontaneous firing after electroporation, right column, calibration pulses of $1 \mathrm{mV} 20 \mathrm{~ms}$.

the surface of the gM $\mu \mathrm{E}$ cap only during sectioning of the Agar block or the observations rather than at earlier stages of the procedures.

\section{FROM EXTRACELLULAR FIELD POTENTIALS TO ACTION POTENTIALS BY ELECTROPORATION AND BACK}

As shown in the experiments of Figures 2-5, prior to electroporation the recorded FPs are composed of a positive phase representing outward current generated by neighboring myocytes followed by a negative phase which represent the voltage-dependent inward currents. The "pure" biphasic potentials recorded by individual $\mathrm{gM} \mu \mathrm{E}$ is consistent with the structural relationships of an engulfed $\mathrm{gM} \mu \mathrm{Es}$ by a single $\mathrm{CM}$. This structural configuration leads to partial electrical "isolation" of one gM $\mathrm{E}$ from the others. Comparison of the FPs recorded by different gM $\mu$ Es revealed some variability in their shapes. This variability is attributed to differences in the seal resistances formed by individual cells and the electrode, and the level of electrical coupling of one cell to the others. A clear delay in the onset of the FPs recorded by the different electrodes reflects the conduction velocity of the APs along the electrically coupled CM network (Figure 4).
After electroporation, which gain Ohmic access to the cell interior, the recorded APs last longer and are significantly larger than the extracellular FPs (which depict the first derivative of the AP voltage over time). The attenuation of the APs amplitude by the system is attributed to the relatively low seal resistance formed between the plasma membrane of the $\mathrm{CM}$ and the laminin coated $\mathrm{gM} \mu \mathrm{E}$ device (Fromherz, 2006; Hai et al., 2010a,b). It is important to note that the shape and amplitude of intracellularly recorded signals by the gM $\mu$ Es-MEA and the AC amplifier used are expected to differ from those recorded by the DC coupled intracellular electrode. The expected differences are related to the impedance generated by the ionic bilayer formed at the interface between the $\mathrm{gM \mu \textrm {E }}$ and the culture medium (Mortari et al., 2007) and the AC amplifier used. Since the parameters that represent individual cell-gM $\mu \mathrm{E}$ junction are not identical, the alterations in the signal shape and attenuation factor are expected to differ for individual $\mathrm{gM} \mu \mathrm{E}$. Nevertheless, these individual alterations can be corrected in future development of the system using the calibration pulse as a reference.

\section{TRANSIENT ELECTROPORATION}

With time after electroporation, the nanopores generated by the electroporating pulse recover (Kinosita and Tsong, 1977; Powell et al., 1986; Tsong, 1991; Freeman et al., 1994). This process is associated with gradual reduction in the amplitude of the APs yet, their shape is maintained quite constant for as long as their amplitude is larger than that of the FPs (Figures 2, 3). The transition between intracellular recordings of APs to that of extracellular FPs is smooth and continuous. As the resistance of the electroporated membrane increases the amplitude of the intracellular component decreases and that of the FP becomes more noticeable (Figure 2). These cascades of events suggest that focal $\mathrm{CM}$ electroporation by micrometer size $\mathrm{gM} \mu \mathrm{E}$ provides transient Ohmic access to the cells interior and that the recovery process exclude the $\mathrm{gM} \mu \mathrm{E}$ from the cell. Since the biphasic shape of the FP recovers it is conceivable to assume that the extracellular space between the CM membrane and the electrode is not dramatically altered. This conclusion is also supported by the observation that a single $\mathrm{CM}$ can be repeatedly penetrated over times. Future studies will be devoted to improve the seal resistance formed between cultured $\mathrm{CM}$ and $\mathrm{gM} \mu \mathrm{E}$ by the functionalization of the $\mathrm{gM \mu E}$ with a membrane like lipid coating (Almquist and Melosh, 2010; Duan et al., 2012). This is expected to improve the duration and amplitude of the recorded APs. In future development of our approach it will be interesting to consider the benefits of being able to gain transient intracellular excess to CM rather than a "permanent penetration into the cells." Long-term microelectrode penetration may eventually rupture the plasma membrane due to friction between the rigid electrodes and beating myocytes.

\section{CONCLUSIONS}

In conclusion, we demonstrated that the gold mushroom-shapedbased MEA has the potential to serve as a convenient tool for repeated, simultaneous, intracellular recordings from an unlimited number of beating CMs. This study together with recent "proof of concept" demonstrations of multiunit, long-term, 
intracellular recordings from excitable cells by micro-(Hai et al., 2010a,b; Fendyur et al., 2011) and nano-MEAs (Tian et al., 2010; Duan et al., 2012; Robinson et al., 2012; Xie et al., 2012) opens up exciting opportunities for basic research and medical applications.

\section{REFERENCES}

Almquist, B. D., and Melosh, N. A. (2010). Fusion of biomimetic stealth probes into lipid bilayer cores. Proc. Natl. Acad. Sci. U.S.A. 107, 5815-5820.

Banach, K., Halbach, M. D., Hu, P., Hescheler, J., and Egert, U. (2003). Development of electrical activity in cardiac myocyte aggregates derived from mouse embryonic stem cells. Am. J. Physiol. Heart Circ. Physiol. 284, H2114-H2123.

Duan, X., Gao, R., Xie, P., CohenKarni, T., Qing, Q., Choe, H. S., Tian, B., Jiang, X., and Lieber, C. M. (2012). Intracellular recordings of action potentials by an extracellular nanoscale field-effect transistor. Nat. Nanotechnol. 7, 174-179.

Fendyur, A., Mazurski, N., Shappir, J., and Spira, M. E. (2011). Formation of essential ultrastructural interface between cultured hippocampal cells and gold mushroom-shaped MEA-toward "IN-CELL" recordings from vertebrate neurons. Front. Neuroeng. 4:14. doi: 10.3389/fneng. 2011.00014

Freeman, S. A., Wang, M. A., and Weaver, J. C. (1994). Theory of electroporation of planar bilayer membranes: predictions of the aqueous area, change in capacitance, and pore-pore separation. Biophys. J. 67, 42-56.

Fromherz, P. (2006). Three levels of neuroelectronic interfacing: silicon chips with ion channels, nerve cells, and brain tissue. Ann. N.Y. Acad. Sci. 1093, 143-160.

Hai, A., Dormann, A., Shappir, J., Yitzchaik, S., Bartic, C., Borghs, G., Langedijk, J. P., and Spira, M. E. (2009a). Spine-shaped gold protrusions improve the adherence and electrical coupling of neurons with the surface of microelectronic devices. J. R. Soc. Interface 6, 1153-1165.

Hai, A., Kamber, D., Malkinson, G., Erez, H., Mazurski, N., Shappir, J., and Spira, M. E. (2009b). Changing gears from chemical adhesion of cells to flat substrata toward engulfment of micro-protrusions by active mechanisms. J. Neural Eng. 6, 066009.

Hai, A., Shappir, J., and Spira, M. E. (2010a). In-cell recordings by extracellular microelectrodes. Nat. Methods 7, 200-202.

Hai, A., Shappir, J., and Spira, M. E. (2010b). Long-term, multisite, parallel, in-cell recording and stimulation by an array of extracellular microelectrodes. J. Neurophysiol. 104, 559-568.

Halbach, M., Egert, U., Hescheler, J., and Banach, K. (2003). Estimation of action potential changes from field potential recordings in multicellular mouse cardiac myocyte cultures. Cell. Physiol. Biochem. 13, 271-284.

Herron, T. J., Lee, P., and Jalife, J. (2012). Optical imaging of voltage and calcium in cardiac cells and tissues. Circ. Res. 110, 609-623.

Itzhaki, I., Maizels, L., Huber, I., ZwiDantsis, L., Caspi, O., Winterstern, A., Feldman, O., Gepstein, A., Arbel, G., Hammerman, H., Boulos, M., and Gepstein, L. (2011). Modelling the long QT syndrome with induced pluripotent stem cells. Nature 471, 225-229.

Jones, I. L., Livi, P., Lewandowska, M. K., Fiscella, M., Roscic, B., and Hierlemann, A. (2011). The potential of microelectrode arrays and microelectronics for biomedical research and diagnostics. Anal. Bioanal. Chem. 399, 2313-2329.

Kinosita, K. Jr., and Tsong, T. Y. (1977). Formation and resealing of pores of controlled sizes in human erythrocyte membrane. Nature 268, 438-441.

Law, J. K., Yeung, C. K., Li, L., Rudd, (2012). The use of SU-8 topographically guided microelectrode array in measuring extracellular field potential propagation. Ann. Biomed. Eng. 40, 619-627.

Matsa, E., Rajamohan, D., Dick, E., Young, L., Mellor, I., Staniforth, A., and Denning, C. (2011). Drug evaluation in cardiomyocytes derived from human induced pluripotent stem cells carrying a long QT syndrome type 2 mutation. Eur. Heart J. $32,952-962$.

Mortari, A., Maaroof, A., Martin, D., and Cortie, M. B. (2007). Mesoporous gold electrodes for sensors based on electrochemical double layer capacitance. Sens. Actuators B Chem. 123, 262-268. J. A., Ingebrandt, S., and Chan, M.

\section{ACKNOWLEDGMENTS}

Parts of this study were carried out at the Charles E. Smith and Prof. Elkes' Laboratory for Collaborative Research in Psychobiology. We thank Noa Mazurski, Nava Shmoel, and Naomi Feinstein for their technical assistance.

Nicolelis, M. A., and Lebedev, M. A. (2009). Principles of neural ensemble physiology underlying the operation of brain-machine interfaces. Nat. Rev. Neurosci. 10, 530-540.

Nygren, A., Kondo, C., Clark, R. B., and Giles, W. R. (2003). Voltage-sensitive dye mapping in Langendorff-perfused rat hearts. Am. J. Physiol. Heart Circ. Physiol. 284, H892- H902.

Omura, Y. (1970). Relationship between transmembrane action potentials of single cardiac cells and their corresponding surface electrograms in vivo and in vitro, and related electromechanical phenomena. Trans. N.Y. Acad. Sci. 32 874-910.

Powell, K. T., Derrick, E. G., and Weaver, J. C. (1986). A quantitative theory of reversible electrical breakdown in bilayer membranes. Bioelectrochem. Bioenerg. 15, 243.

Purves, R. D. (1981). Microelectrode Methods for Intracellular Recording, and Ionophoresis. London, New York: Academic Press

Reppel, M., Pillekamp, F., Brockmeier, K., Matzkies, M., Bekcioglu, A. Lipke, T., Nguemo, F., Bonnemeier, H., and Hescheler, J. (2005). The electrocardiogram of human embryonic stem cell-derived cardiomyocytes. J. Electrocardiol. 38, 166-170.

Robinson, J. T., Jorgolli, M., Shalek, A. K., Yoon, M. H., Gertner, R. S., and Park, H. (2012). Vertical nanowire electrode arrays as a scalable platform for intracellular interfacing to neuronal circuits. Nat. Nanotechnol. 7, 180-184.

Sakmann, B., and Neher, E. (1984) Patch clamp techniques for studying ionic channels in excitable membranes. Annu. Rev. Physiol. 46, 455-472.

Sanchez-Bustamante, C. D., Frey, U., Kelm, J. M., Hierlemann, A., and Fussenegger, M. (2008). Modulation of cardiomyocyte electrical properties using regulated bone morphogenetic protein-2 expression. Tissue Eng. Part A 14, 1969-1988.

Sekine, H., Shimizu, T., Dobashi, I., Matsuura, K., Hagiwara, N., Takahashi, M., Kobayashi, E., Yamato, M., and Okano, T. (2011). Cardiac cell sheet transplantation improves damaged heart function via superior cell survival in comparison with dissociated cell injection. Tissue Eng. Part A 17, 2973-2980.

Shneyvays, V., Nawrath, H., Jacobson, K. A., and Shainberg, A. (1998). Induction of apoptosis in cardiac myocytes by an A3 adenosine receptor agonist. Exp. Cell Res. 243, 383-397.

Spira, M. E., Kamber, D., Dormann, A., Cohen, A., Bartic, C., Borghs, G., Langedijk, J. P. M., Yitzchaik, S., Shabthai, K., and Shappir, J. (2007). "Improved neuronal adhesion to the surface of electronic device by engulfment of protruding micronails fabricated on the chip surface," in Transducers ' 07 and Eurosensors Xxi, Digest of Technical Papers, Vols. 1 and 2, (Lyon, France: Institute of Electrical and Electronics Engineers (IEEE)), U628.2616-U629.2616.

Spira, M. E., Oren, R., Dormann, A., and Gitler, D. (2003). Critical calpain-dependent ultrastructural alterations underlie the transformation of an axonal segment into a growth cone after axotomy of cultured Aplysia neurons. J. Comp. Neurol. 457, 293-312.

Studer, D., Humbel, B. M., and Chiquet, M. (2008). Electron microscopy of high pressure frozen samples: bridging the gap between cellular ultrastructure and atomic resolution. Histochem. Cell Biol. 130, 877-889.

Tian, B., Cohen-Karni, T., Qing, Q., Duan, X., Xie, P., and Lieber, C. M. (2010). Three-dimensional, flexible nanoscale field-effect transistors as localized bioprobes. Science 329 , 830-834.

Tsong, T. Y. (1991). Electroporation of cell membranes. Biophys. J. 60, 297-306.

Wang, P. Y., Yu, J., Lin, J. H., and Tsai, W. B. (2011). Modulation of alignment, elongation and contraction of cardiomyocytes through a combination of nanotopography and rigidity of substrates. Acta Biomater. 7, 3285-3293.

Xie, C., Lin, Z., Hanson, L., Cui, Y., and Cui, B. (2012). Intracellular recording of action potentials by nanopillar electroporation. Nat. Nanotechnol. 7, 185-190.

Yeung, C. K., Sommerhage, F., Wrobel, G., Law, J. K., Offenhausser, A. 
Rudd, J. A., Ingebrandt, S., and Chan, M. (2009). To establish a pharmacological experimental platform for the study of cardiac hypoxia using the microelectrode array. J. Pharmacol. Toxicol. Methods 59, 146-152.

Zipes, D. P., and Jalife, J. (2009). Cardiac Electrophysiology: From Cell to Bedside. Philadelphia, PA: W.B. Saunders Company.

Conflict of Interest Statement: The authors declare that the research was conducted in the absence of any commercial or financial relationships that could be construed as a potential conflict of interest.
Received: 25 June 2012; accepted: 05 August 2012; published online: 24 August 2012.

Citation: Fendyur $A$ and Spira ME (2012) Toward on-chip, in-cell recordings from cultured cardiomyocytes by arrays of gold mushroom-shaped microelectrodes. Front. Neuroeng. 5:21. doi: 10.3389/fneng.2012.00021
Copyright (c) 2012 Fendyur and Spira. This is an open-access article distributed under the terms of the Creative Commons Attribution License, which permits use, distribution and reproduction in other forums, provided the original authors and source are credited and subject to any copyright notices concerning any third-party graphics etc. 


\section{APPENDIX}

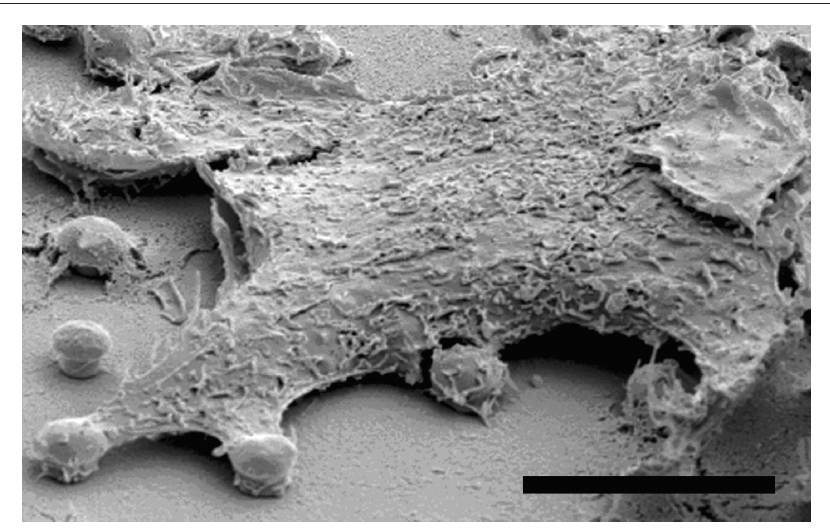

FIGURE A1 | A scanning electron micrograph depicting the relationships between a cultured cardiomyocyte and $\mathrm{gM} \mu \mathrm{Es}$. Calibration: $10 \mu \mathrm{m}$. 